\title{
DENSIFICATION SUPERFICIELLE DE MATÉRIAUX POREUX PAR CHOC LASER
}

\author{
D. ZAGOURI*, J.-P. ROMAIN**, B. DUBRUJEAUD ${ }^{* * *}$ et M. JEANDIN*** \\ ${ }^{*}$ Peugeot S.A. Etudes et Recherches, D.R.A.S. . Route de Gizy, \\ F-78140 Vélizy, France \\ ** Laboratoire d'Energétique et de Détonique, URA-CNRS 193. \\ ENSMA, F-86034 Poitiers, France \\ *** Centre des Matériaux Pierre-Marie Fourt, Ecole des Mines de \\ Paris, BP. 87, F-91003 Evry, France
}

\begin{abstract}
Résumé - Les chocs laser permettent d'obtenir une densification superficielle sur un matériau poreux. La profondeur affectée est de l'ordre de quelques centaines de microns. Cette étude présente les résultats numériques obtenus avec un code hydrodynamique monodimensionnel lagrangien décrivant l'évolution d'une cible soumise d un choc laser. Le comportement des matériaux poreux est modélisé en utilisant le principe du modele $P$ - $\alpha$ de Herrmann. Le code nous permet d'obtenir le profil de densification dans la cible en fonction des conditions d'irradiation, de la nature du matériau et de sa porosité initiale. Des expériences ont été réalisées sur des échantillons de poudre d'aluminium et d'acier fritté (Distaloy $A E$ ). La configuration de plasma confiné a été utilisée : la cible est recouverte par un milieu transparent au rayonnement incident, ce procédé augmente la pression et sa durée de maintien par rapport à une irradiation directe. Des échantillons ont été observés en microscopie optique. Le profil de porosité résiduelle est estimé par analyse d'image. Les résultats obtenus avec le code sont en bon accord avec les résultats expérimentaux.
\end{abstract}

\begin{abstract}
Laser-driven shock-waves are used as a surface treatement for compacting porous materials. The compaction depth is typically a few hundreds microns. The behavior of the porous medium is described through a compaction model based on the $P$ - $\alpha$ theory of Herrmann. This model has been introduced into a one-dimensional finite difference hydrodynamic code describing the behavior of a target under the action of a laser-generated shock-wave. The code enables us to compute the compaction depth as a function of irradiation conditions, nature and initial porosity of the material. Experiments are performed on aluminum powder and sintered porous steel (Distaloy $A E$ ). The samples are covered with a transparent window acting as a confinement for the plasma generated by absorption of the incident radiation on the target surface. This process increases both the pressure and the duration of the applied pressure in comparison with a bare target irradiation. Samples are observed by optical microscopy. The residual porosity is estimated by image analysis. Experimental results and computed compaction profiles correlate well.
\end{abstract}

\section{1.- Introduction}

Le compactage dynamique des matériaux poreux est couramment réalisé au moyen de générateurs de choc classiques tels que explosifs ou impacts de projectile. Dans ce cas, le temps de maintien de la pression permet à l'onde de choc de se propager sur des distances suffisantes pour obtenir le compactage de pièces massives. Au contraire, le temps de maintien très court des chocs laser (1$100 \mathrm{~ns}$ ) conduit à une densification sur des profondeurs de l'ordre de quelques centaines de microns 11/. Ce procédé constitue donc un traitement de surface.

Cette étude a pour but de caractériser les épaisseurs de compactage susceptibles d'être obtenues par choc laser en fonction de la nature du matériau, de sa porosité initiale et des conditions d'irradiation. 
A cet effet, un modèle de comportement des matériaux poreux a été développé et introduit dans un code de calcul permettant de décrire l'évolution des paramètres caractérisant une cible soumise à un choc laser.

Des expériences ont été réalisées sur deux matériaux (aluminium et acier). Les effets de compactage obtenus sont comparés aux résultats calculés par simulations numériques.

\section{2.- Modélisation des matériaux poreux}

Un modèle simple, négligeant le travail nécessaire à la fermeture des pores, a tout d'abord été utilisé. Ce modèle donne des résultats très satisfaisants pour des pressions suffisament élevées (supérieures à environ $100 \mathrm{kbar}$ ) $/ 2 /$. Cependant, l'hypothèse de non rigidité implique qu'une pression quasi-nulle suffit pour permettre au matériau de passer du volume initial $\mathrm{V}_{\mathrm{o}}$ au volume du compact correspondant $\mathrm{Vs}_{\mathrm{o}}$ (le compactage total est ainsi atteint); Il ne permet donc pas de décrire un état de compactage partiel.

Nous avons développé un second modèle en utilisant le principe de comportement des matériaux poreux introduit par W. Herrmann /3/. Ce principe, largement admis, se caractérise par trois points :

- L'utilisation d'une équation d'état des matériaux compacts pour la fraction solide du matériau poreux (grandeurs caractéristiques : Vs, Ps, Es) :

$$
P s=f(V s, E s)
$$

L'équation d'état de Mie-Gruneïsen référencée par l'Hugoniot du matériau compact a été utilisée.

- Deux hypothèses sur les relations entre les grandeurs caractéristiques du matériau poreux $(\mathrm{V}, \mathrm{P}, \mathrm{E})$ et les grandeurs caractéristiques de sa fraction solide :

$$
\begin{aligned}
& E=\text { Es (énergie de surface négligée ) } \\
& P=\frac{P s}{\alpha} \text { (relation proposée par M. M. Carol \& A. C. Holt /4/) } \\
& \text { où } \alpha=\frac{V}{V S}
\end{aligned}
$$

On obtient alors l'équation d'état du matériau poreux à partir de celle du matériau compact :

$$
P=\frac{f\left(\frac{V}{\alpha}, E\right)}{\alpha}
$$

- Une relation décrivant les variations du degré de compactage $\alpha$ est nécessaire. La loi de compactage en fonction du volume $\alpha(V)$ proposée par D. E. Maxwell /5/ a été préférée à la relation polynomiale en fonction de la pression $\alpha(\mathrm{P})$ utilisé par W. Herrmann, en raison de la facilité de mise en place dans le code de calcul d'une relation fonction du volume.

Le modèle prend en compte une phase initiale élastique pendant laquelle le degré de compactage varie peu.

Pour la phase de compactage, un paramètre $\beta$ permet d'ajuster la position de la courbe de Hugoniot en fonction de l'aptitude du matériau poreux à être compacté. Toutes les courbes tendent, à haute pression vers l'Hugoniot du matériau poreux sans rigidité pour lequel le compactage est total $(\alpha=1)$. On peut observer l'influence du paramètre $\beta$ sur la figure 1 . 
Ce modèle a été introduit dans un code de calcul monodimensionel hydrodynamique Lagrangien qui calcule l'évolution des variables thermodynamiques et cinétiques au cours de la propagation d'un choc dans une cible. Ce code permet en particulier d'obtenir les profils de pression et de compactage dans la cible à différents instants.

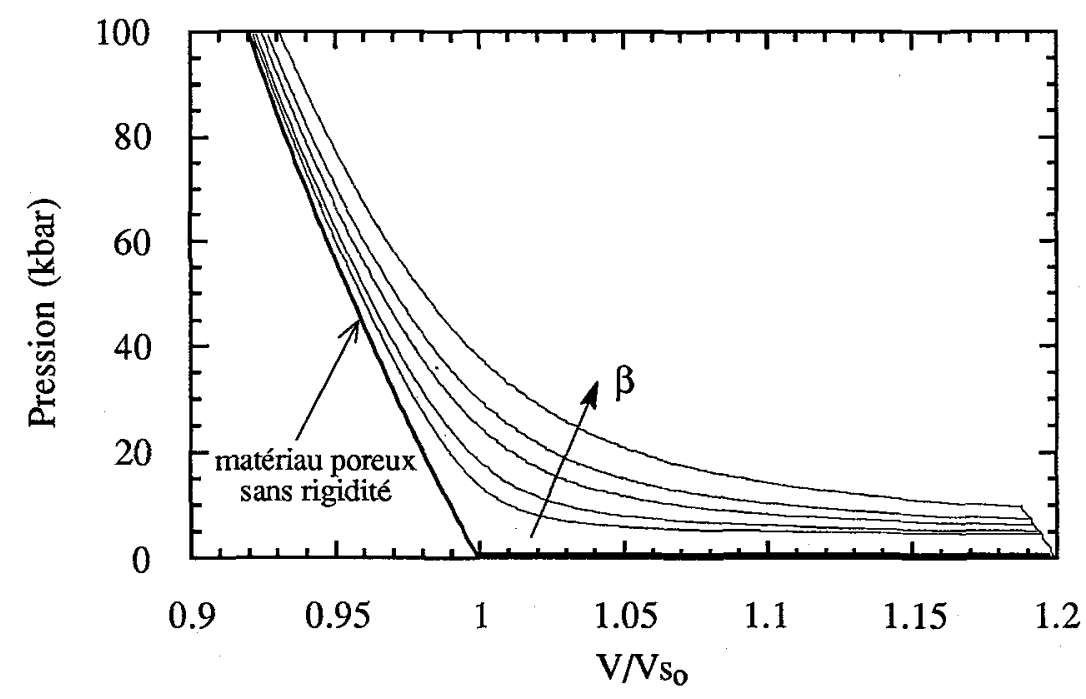

Figure 1

Courbes de Hugoniot pour l'aluminium de porosité initiale $\alpha_{0}=1.2$.

Influence du paramètre $\beta$.

\section{3.- Expériences}

Les expériences ont été réalisées sur le laser verre phosphate-néodyme du laboratoire L.U.L.I. (Ecole Polytechnique).

Deux matériaux ont été utilisés pour les expériences de compactage :

- Une poudre d'aluminium à grains sphériques de diamètre compris entre 10 et $100 \mu \mathrm{m}$, comprimée uniaxialement à froid, de volume spécifique supérieur d'environ $22 \%$ à celui de l'aluminium compact $\left(\alpha_{0}=1.22\right)$

- Un acier pré-allié Distaloy $\mathrm{AE}(\mathrm{Ni} 5 \%, \mathrm{Cu} 2 \%, \mathrm{C} 0.6 \%)$ comprimée uniaxialement et fritté à $1120^{\circ} \mathrm{C}$ pendant 30 minutes, de volume spécifique supérieur d'environ $8.5 \%$ à celui de l'acier compact $\left(\alpha_{0}=1.085\right)$

Le choc est généré par focalisation d'une impulsion laser sur la surface de l'échantillon de manière à former un plasma à haute pression. L'interaction est réalisée en configuration confinée (figure 2) c'est-à-dire en recouvrant la cible par un matériau transparent au rayonnement laser, cette méthode permet d'augmenter sensiblement la pression et son temps de maintien par rapport à une irradiation directe, procurant ainsi des conditions plus favorables pour le compactage.

Les effets thermiques en surface des échantillons sont évités en intercalant entre la cible et le confinement une feuille métallique mince ou en recouvrant la cible par une couche de peinture absorbante. 


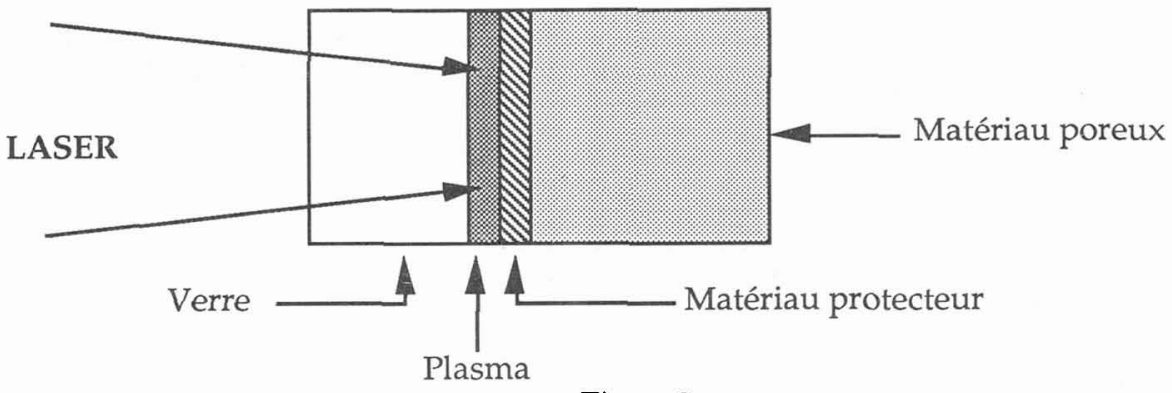

Figure 2

Configuration de tir en interaction confinée.

\section{4.- Analyse des résultats}

- Un échantillon de poudre d'aluminium a été irradié dans les conditions suivantes :

- longueur d'onde : $\lambda=1.06 \mu \mathrm{m}$

- énergie : $\mathrm{E}=21.5 \mathrm{~J}$

- durée à mi-hauteur de l'impulsion : $\tau=8.8 \mathrm{~ns}$, impulsion semi-gausienne

- flux incident $: \mathrm{I}=8.6 \mathrm{GW} / \mathrm{cm}^{2}$

La cible était protégée par une feuille d'aluminium d'épaisseur $40 \mu \mathrm{m}$.

Compte tenu de l'impédance de choc du verre et de l'aluminium et en considérant le plasma comme un gaz parfait absorbant le rayonnement laser avec un rendement de $16 \%$, on peut évaluer le profil de pression appliqué sur la cible (figure 3) $/ 6 / \pi /$.

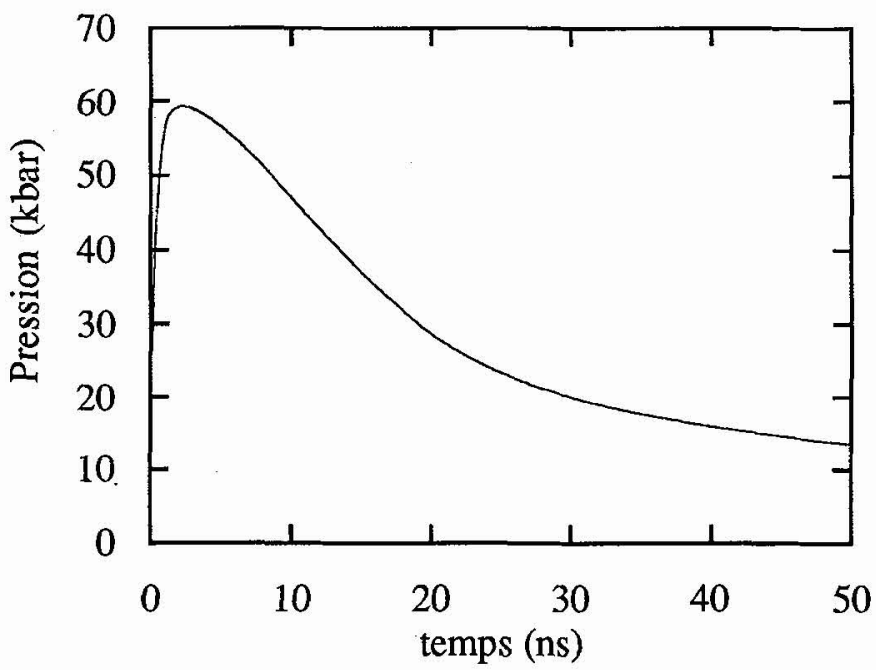

Figure 3

Profil de pression appliqué sur l'échantillon de poudre d'aluminium. 
Une coupe de l'échantillon ayant subi le traitement est representée sur la figure 4 : on constate une densification totale sur une profondeur d'environ $100 \mu \mathrm{m}$ suivi d'un gradient de compactage. On peut remarquer que la feuille d'aluminium a été plaquée sur la poudre.

Le degré de porosité résiduelle $\frac{\mathrm{V}-\mathrm{V} \mathrm{s}_{\mathrm{O}}}{\mathrm{V} \mathrm{s}_{\mathrm{o}}}=\alpha-1$ a été quantifié par analyse d'image; les points obtenus figurent sur le graphique placé en correspondance avec la micrographie.

La courbe calculée à l'aide du code est également représentée sur ce graphique. On constate un bon accord entre les résultats théoriques et les résultats expérimentaux.
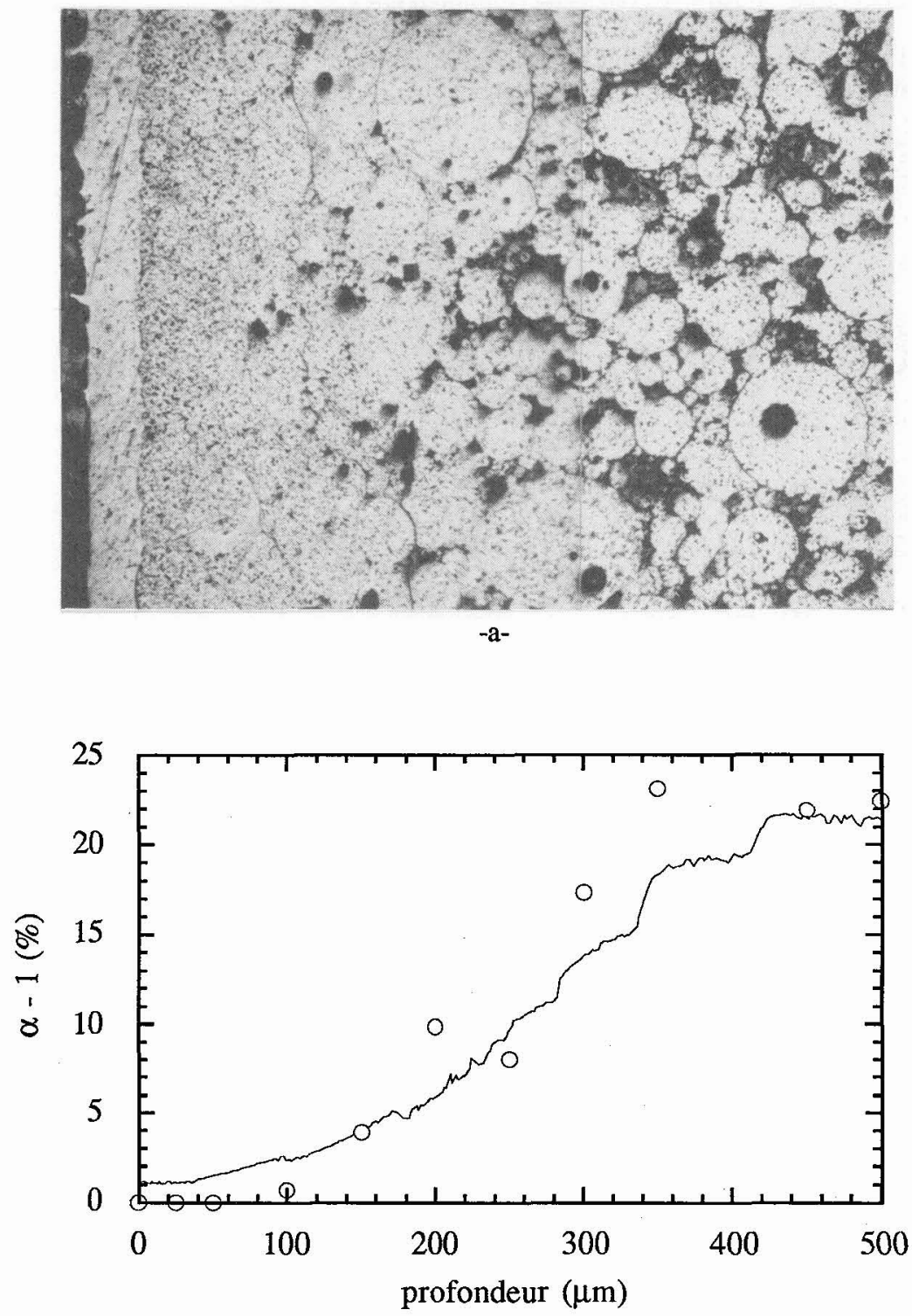

-b-

Figure 4

Coupe de l'échantillon de poudre d'aluminium après choc (a).

Profil de compactage calculé et points expérimentaux obtenus par analyse d'ímage (b). 
- L'irradiation d'un échantillon d'acier fritté a eu lieu dans les conditions suivantes :

$$
\begin{aligned}
& -\lambda=1.06 \mu \mathrm{m} \\
& -\mathrm{E}=51 \mathrm{~J} \\
& -\tau=28 \mathrm{~ns} \text { Impulsion semi-gausienne } \\
& -\mathrm{I}=5.5 \mathrm{GW} / \mathrm{cm}^{2}
\end{aligned}
$$

La cible était protégée par une couche de peinture noire.

Le profil de pression appliqué sur la cible a été évalué de la même manière que pour l'échantillon précédent. Il est représenté sur la figure 5.

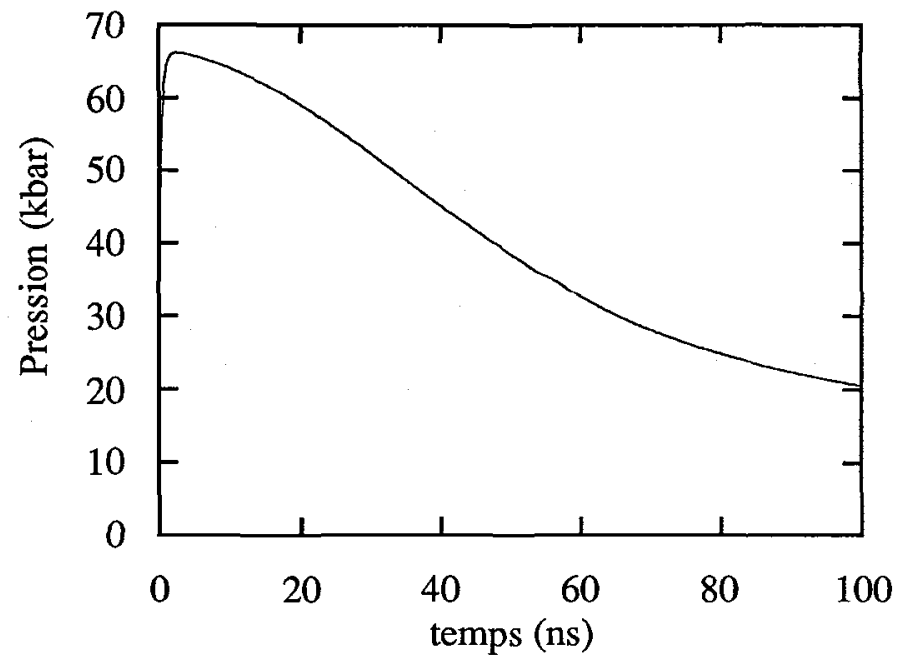

Figure 5

Profil de pression appliqué sur l'échantillon d'acier fritté.

Une coupe de l'échantillon traité est représentée sur la figure 6:On constate que la densification totale n'est pas atteinte, même aux faibles profondeurs (degré de porosité résiduel supérieur à $3 \%$ ).

La présence de porosités résiduelles de taille très variable est la cause d'une dispersion importante pour les points obtenus par analyse d'image, l'accord des résultats expérimentaux avec la courbe de degré de porosité résiduel calculée avec le code est néammoins satisfaisant. 

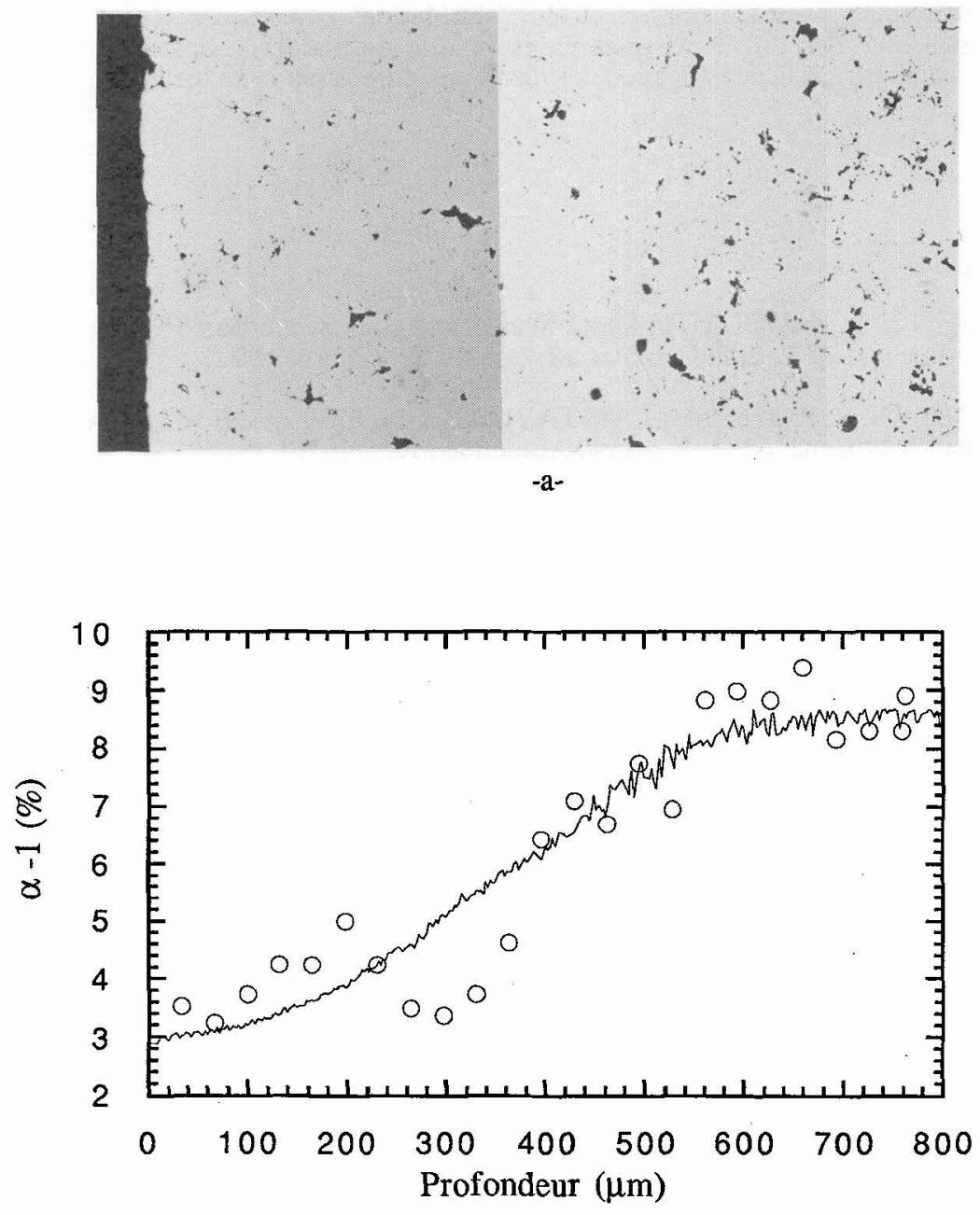

$-b-$

Figure 6

Coupe de l'échantillon d'acier fritté après choc (a).

Profil de compactage calculé et points expérimentaux obtenus par analyse d'image (b).

\section{5.- Conclusion}

Les résultats obtenus confirment la possibilité de réaliser par choc laser une densification superficielle de matériaux poreux.

L'étude a été menée sur deux matériaux métallurgiquement très différents : une poudre d'aluminium et un acier fritté. Du point de vue de la densification, on peut constater d'abord que pour des pressions de chocs voisines (de l'ordre de $60 \mathrm{kbar}$ ), un compactage total est obtenu sur l'aluminium et un compactage seulement partiel sur l'acier fritté. D'autre part les épaisseurs de matériau affectées par le choc (environ $400 \mu \mathrm{m}$ pour l'aluminium et environ $550 \mu \mathrm{m}$ pour l'acier) sont du même ordre de grandeur alors que les caractéristiques d'irradiation étaient très différentes, en particulier la durée de l'impulsion laser. 
Ces deux exemples illustrent la diversité de comportement des matériaux poreux sous choc de courte durée. Des expériences complémentaires et leurs simulations numériques seront nécessaire pour déterminer l'influence des différents paramètres (nature du matériau, degré de porosité initial, conditions d'irradiation) sur la densification des matériaux poreux par cette méthode.

\section{Références}

/1/ J.-P. ROMAIN \& D. ZAGOURI, in Shock Waves and High Strain Rate Phenomena, eds. M. A. Meyers, L. E. Murr and K. P. Staudhammer, M. Dekker New York (1991)

/2/ R. G. MCQUEEN, S. P. MARSH, J. W. TAYLOR, J. N. FRITZ, and W. J. CARTER in HighVelocity Impact Phenomena, ed. R. Kinslow, Academic Press (1970)

/3/ W. HERRMANN, J. Appl. Phys. 40, 3490 (1969)

14/ M. M. CARROLL \& A. C. HOLT, J. Appl. Phys. 43, 759 (1972)

15/ D. E. MAXWELL, Science Applications, San Leandro, SATR 82-4 (1982)

/6/ P. DARQUEY, J.-P. ROMAIN, J. Appl. Phys. 68, 1926 (1990)

77/ J. FOURNIER, R. FABBRO, P. BALLARD, J. VIRMONT, Proceedings, $19^{\text {th }}$ ECLIM, Madrid (1988) 